\title{
First-line treatment selection with organoids of an EGFRm + TP53m stage IA1 patient with early metastatic recurrence after radical surgery and follow-up
}

\author{
Ziqi Jia ${ }^{1,2 \#}$, Yanyu Wang ${ }^{1 \#}$, Lei Cao ${ }^{1 \#}$, Yadong Wang ${ }^{1}$, Yang Song ${ }^{1}$, Xiaoying Yang ${ }^{1,2}$, Zhongxing Bing ${ }^{1}$, \\ Zhili Cao ${ }^{1}$, Peng Liu ${ }^{3}$, Shuyang Zhang ${ }^{3,4}$, Zexin Chen ${ }^{5}$, Min Huang ${ }^{5}$, Yan Yu ${ }^{5}$, Han Han-Zhang ${ }^{6}$, \\ Jinlei Song ${ }^{6}$, Daniel C. Christoph ${ }^{7}$, Antonio Passaro ${ }^{8}$, Cesare Gridellii, Tomoyuki Hishida ${ }^{10}$, \\ Naixin Liang ${ }^{1}$, Shanqing Li $^{1}$
}

${ }^{1}$ Department of Thoracic Surgery, Peking Union Medical College Hospital, Chinese Academy of Medical Sciences, Beijing, China; ${ }^{2}$ Eight-Year MD Program, Peking Union Medical College, Chinese Academy of Medical Sciences, Beijing, China; ${ }^{3}$ Medical Research Center, Central Laboratory, Peking Union Medical College Hospital, Chinese Academy of Medical Sciences, Beijing, China; ${ }^{4}$ Department of Cardiology, Peking Union Medical College Hospital, Chinese Academy of Medical Sciences, Beijing, China; ${ }^{5}$ Accurate International Biotechnology Co. Ltd., Guangzhou, China; ${ }^{6}$ Burning Rock Biotechnology Co. Ltd., Guangzhou, China; ${ }^{7}$ Department of Medical Oncology/Haematology, Evang. Kliniken Essen-Mitte, Essen, Germany; ${ }^{8}$ Division of Thoracic Oncology, IEO, European Institute of Oncology IRCCS, Milan, Italy; ${ }^{9}$ Division of Medical Oncology, "S.G. Moscati" Hospital, Contrada Amoretta, 83100, Avellino, Italy; ${ }^{10}$ Division of Thoracic Surgery, Department of Surgery, Keio University School of Medicine, Tokyo, Japan

\#These authors contributed equally to this work.

Correspondence to: Naixin Liang, MD; Shanqing Li, MD. Department of Thoracic Surgery, Peking Union Medical College Hospital, Peking Union Medical College and Chinese Academy of Medical Sciences, Beijing 100730, China. Email: pumchnelson@163.com; 1sq6768@sohu.com.

Submitted May 09, 2020. Accepted for publication Jun 02, 2020.

doi: $10.21037 /$ jtd-20-1882

View this article at: http://dx.doi.org/10.21037/jtd-20-1882

\section{Introduction}

Stage I lung adenocarcinoma (LADC) has a 5-year overall survival (OS) of $68 \%$, with stage IA1 reaching as high as $91 \%(1-3)$. Surgery is the conventional treatment and currently preferred local treatment that can achieve complete relief in early-stage non-small cell lung cancer (NSCLC) (4). However, the relapse of some early-stage NSCLC has brought attention to the post-operative management of resectable tumors (5). According to phase III trials, adjuvant chemotherapy after R0 resection may achieve an improvement of $5 \%$ in 5 -year survival, but the result is limited among patients with NSCLC staged higher than IB. In stage IA patients, the use of adjuvant chemotherapy can be even detrimental (6). Moreover, the current clinical practice guideline does not recommend adjuvant chemotherapy in stage IA patients after R0 resection (7). With a low genetic testing rate in earlystage LADC, no evidence from phase III clinical trials has demonstrated that epidermal growth factor receptor- mutated $(E G F R \mathrm{~m})$ stage IA patients can benefit from adjuvant $E G F R$-tyrosine kinase inhibitors (TKIs).

Without adjuvant therapy, $5-10 \%$ patients will suffer from early recurrence $2-3$ years after their curative surgery (8). It remains unclear how to identify the high-risk cohort in stage IA patients. The evaluation on the prognosis of stage IA patients is mainly based on pathological findings: the size of invasive component, lymphatic or vascular invasion, or visceral pleural invasion $(9,10)$. Because genetic testing is mainly performed in advanced stage NSCLC $(11,12)$, the prognostic and predictive values of genomic profiling has not been widely recognized in early-stage $\operatorname{NSCLC}(13,14)$. However, it has been reported that advanced NSCLC patients harboring co-mutation of EGFR and TP53 have a poorer prognosis (15-17). Relapse in resected NSCLC patients is often reported as local or regional recurrence and metastasis. For these advanced stage recurrent patients, there still lacks high-level evidence as to whether these patients should be treated in the same way as advanced stage patients for the first-line treatment, or if they should 
be treated with a more aggressive regiment based on their formal relapsed history. It is also unclear whether these patients can benefit from first-line osimertinib in case of activating EGFR mutation. Yet, according to a multicenter phase III trial of osimertinib used as first-line treatment in EGFRm advanced stage NSCLC (FLAURA), though osimertinib established a substantial improvement in median progression-free survival (PFS) and median OS, which converged down toward the comparing arm of firstgeneration TKI (18). Based on the current evidence, a preclinical model could be applied in treatment selection in personalized precision therapy.

Patient-derived organoids (PDO) are 3D tumor cultures that are grown from tissue-specific stem cells derived from the primary tumor (19-21). They are cultured in a $3 \mathrm{D}$ environment with essential growth factors, collagen, and other extracellular matrix (ECM) components structured by Matrigel. PDO can recapitulate the histological, structural (20), and genetic characteristics (21-23) of the primary tumor. As a preclinical tumor model, PDO has higher similarity than cell line cultures and saves both time and cost compared to a patient-derived xenograft (24). Organoid drug sensitivity assay uses $\mathrm{IC}_{50}$ as an indicator in individual drug screening for a primary tumor. Randomized clinical trials of colon-rectal cancer, breast cancer, and NSCLC are ongoing in Europe to evaluate the recapitulation of primary tumor drug sensitivity (TUMOROID, NL49002.031.14) and the efficacy of last-line drug selection (SENSOR, NL50400.031.14).

Herein, we report a case of a 66-year-old Chinese male who is a former smoker (40 pack-years) with a case of early metastatic recurrence of stage IA1 LADC harboring $E G F R^{\mathrm{L} 858 \mathrm{R}}$ and $T P 53^{\mathrm{R} 110 \mathrm{~L}}$ after $\mathrm{R}_{0}$ resection and the response to firstline therapy osimertinib. An international multidisciplinary team (iMDT) discussion is presented that discusses the future treatment strategies. We present the following case in accordance with the CARE reporting checklist (available at http://dx.doi.org/10.21037/jtd-20-1882). Written informed consent was obtained from the patient for publication of this manuscript and any accompanying images.

\section{Case presentation}

A 66-year-old male was admitted to our hospital for suspicious metastatic recurrence in his post-operative physical examination with no clinical manifestation. He received video-assisted thoracoscopic surgery (VATS) left upper lobectomy and associated hilar and mediastinal lymph nodes dissections in other hospital 3 years ago. His primary tumor was pathologically diagnosed as LADC with immunohistochemistry (IHC) showing CK7(+), TTF(+), Napsin A(-), staged as $\mathrm{T}_{1 \mathrm{a}} \mathrm{N}_{0} \mathrm{M}_{0}$ (stage IA1), with a microscopically negative margin, no invasion of visceral pleura, lymphatic or venous vessels was observed. The diagnosis of metastatic recurrence was based on chest computed tomography (CT), which showed multiple nodules in the right lung (the largest one was sized $1.1 \mathrm{~cm}$ $\times 0.9 \mathrm{~cm}$ ) and an enhanced mass with soft tissue density at the left hilum. ${ }^{18} \mathrm{~F}$-fluorodeoxyglucose positron emission tomography/computed tomography $\left({ }^{18}\right.$ FDG-PET/CT) scan showed a $1-\mathrm{cm}$ nodule in his right supraclavicular fossa with increased avidity and a maximum standard uptake value $\left(\mathrm{SUV}_{\max }\right)$ of 4.2 (Figure 1). The routine laboratory test [including carcinoembryonic antigen (CEA), CYFRA21-1, CA125, alpha-fetoprotein (AFP), CA19-9, CA15-3, CA72-4, total prostate-specific antigen (tPSA), free PSA (fPSA), squamous cell carcinoma antigen (SCC$\mathrm{Ag})$ ] results showed elevations in multiple tumor markers, including tPSA, fPSA, SCC-Ag, and CYFRA21-1. Pelvic CT ruled out the suspicion of prostate cancer or metastasis. The patient presented with no complaint of discomfort or disability, and was subsequently assessed as ECOGPS 0 . The final pathological diagnosis was lymph node metastatic adenocarcinoma by surgical removal of the right supraclavicular lymph nodes (Figure 2). IHC staining was not repeated.

Molecular diagnosis of the patient's formalin-fixed paraffin-embedded (FFPE) tissue specimens based on next-generation sequencing (NGS) showed $E G F R^{\mathrm{L} 858 \mathrm{R}}+$ $T P 53^{\mathrm{R} 110 \mathrm{~L}}$ mutations in his primary tumor, and $E G F R^{\mathrm{L} 858 \mathrm{R}}+$ $T P 53^{\mathrm{R} 110 \mathrm{~L}}+C D K N 2 A^{\mathrm{H} 83 \mathrm{Y}}$ in the lymph nodes. Circulating tumor DNA (ctDNA) of the plasma was also tested, but no mutation was detected.

PDO cultures were successfully established using culture methods described in the supplemental materials (Figure 3A). Efficacy of EGFR-TKI was demonstrated by carrying out cell viability and proliferation assays using the established organoid cultures. Cell viability curves of organoids treated with gefitinib, erlotinib, afatinib, icotinib, and osimertinib are shown in Figure 3B. The results showed that the IC50 of osimertinib was the lowest among the EGFR-TKIs that were tested.

\section{Treatment}

Based on the evidence listed above, the recurrent metastatic 


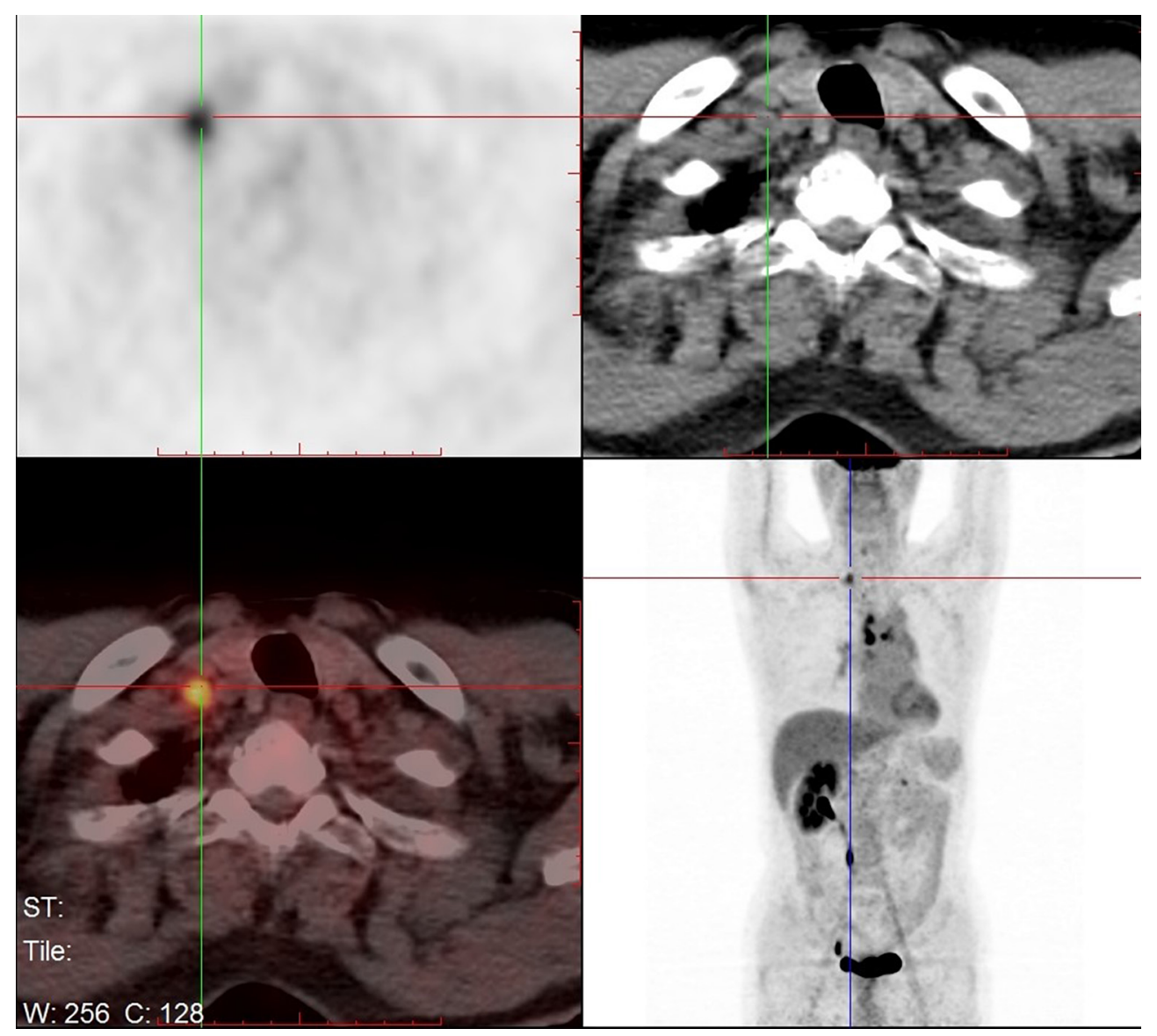

Figure $1{ }^{18} \mathrm{FDG}-\mathrm{PET} / \mathrm{CT}$ scan showing a $1-\mathrm{cm}$ nodule in the right supraclavicular fossa with increased avidity of $\mathrm{SUV}_{\max }=4.2 .{ }^{18} \mathrm{FDG}-$ $\mathrm{PET} / \mathrm{CT},{ }^{18} \mathrm{~F}$-fluorodeoxyglucose positron emission tomography/computed tomography; $\mathrm{SUV}_{\max }$, maximum standard uptake value.

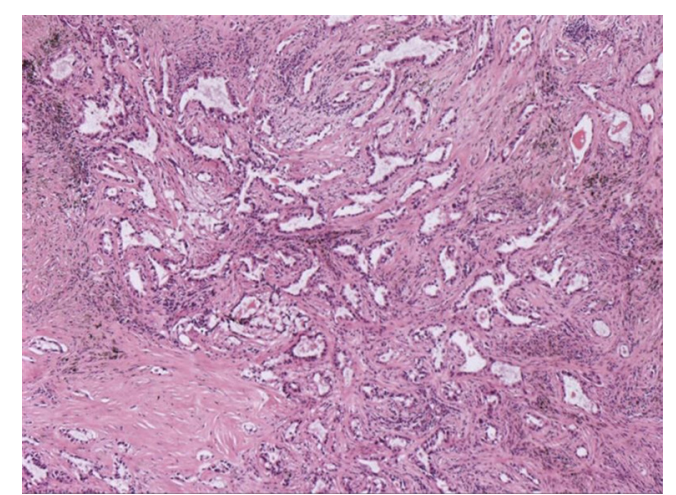

Figure 2 Pathological diagnosis of right supraclavicular lymph nodes as lymph node metastatic adenocarcinoma. HE staining, original magnification $\times 40$. lymph node adenocarcinoma of this patient harbored both $E G F R^{\mathrm{L} 858 \mathrm{R}}$ and $T P 53^{\mathrm{R} 110 \mathrm{~L}}$, and a novel lymph node specific mutation-1 $C D K N 2 A^{\mathrm{H} 83 \mathrm{Y}}$. Additionally, based on the results of the drug response curves of the 5 EGFR-targeted TKIs, osimertinib was suggested to be the most efficacious drug. Thus, the patient began receiving oral osimertinib, $80 \mathrm{mg}$, once daily and achieved a PFS of 9 months (Figure 4).

\section{iMDT discussion}

\section{Department of Oncology}

In this case, osimertinib was chosen as first-line therapy based on the NGS result of the primary tumor and the 

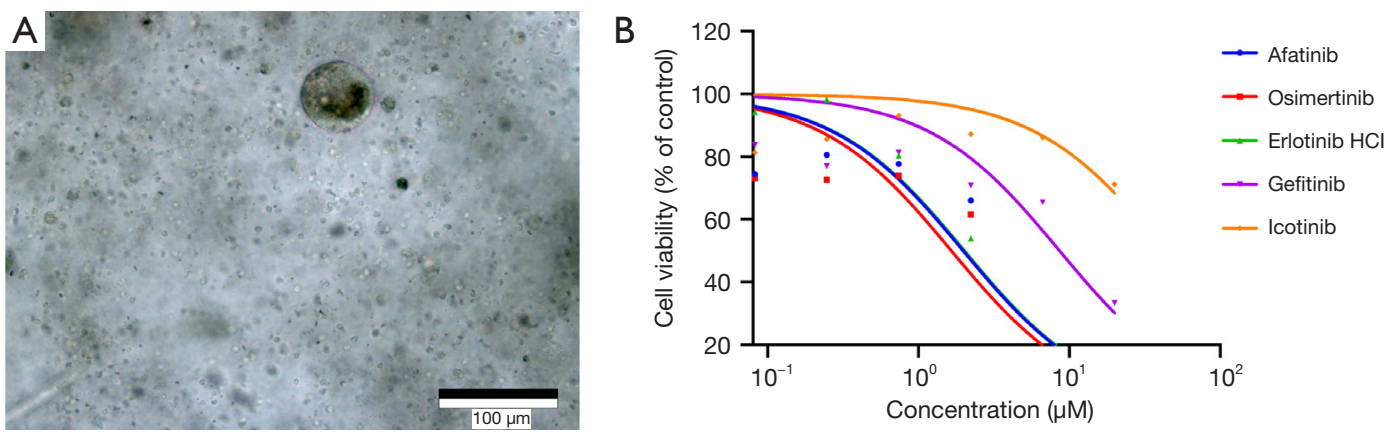

Figure 3 Drug responses of patient-derived lymph node metastatic adenocarcinoma organoids. (A) Organoid culture of lymph node metastatic adenocarcinoma; (B) dose-response curves of patient-derived lymph node metastatic adenocarcinoma organoids treated with EGFR-TKI afatinib, osimertinib, erlotinib $\mathrm{HCl}$, gefitinib, and icotinib. Cell viability was measured by an ATP-based assay after 4 days of drug incubation. TKI, tyrosine kinase inhibitor.
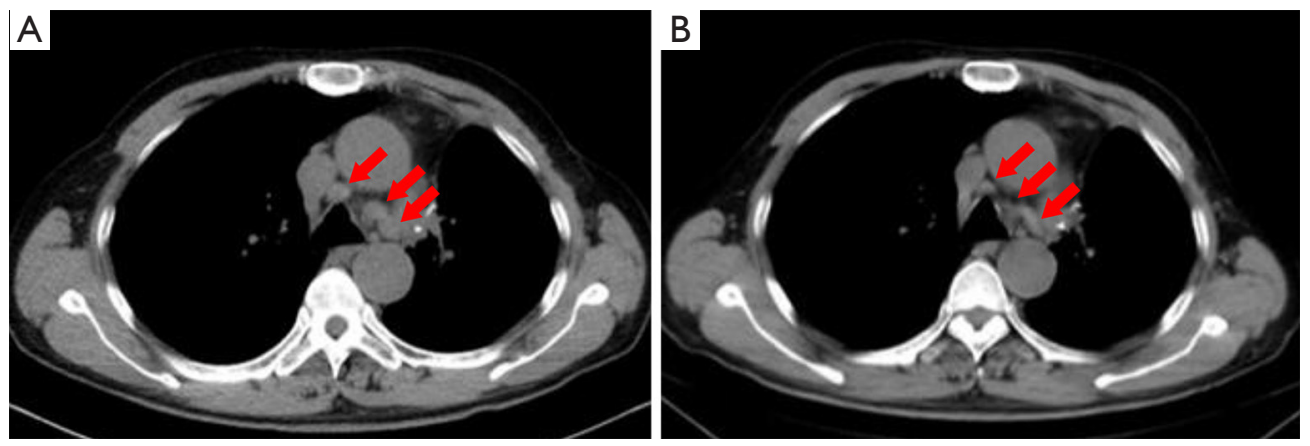

Figure 4 Effect of osimertinib in this patient with multiple mediastinal lymph node metastasis. Computed tomography scan of the chest before (A) and after (B) treatment with osimertinib. Shrinkage of multiple mediastinal lymph nodes (arrows) was observed.

metachronous second primary tumor (MST). According to FLAURA (18), osimertinib used as first-line TKI in EGFRm NSCLC can achieve a median PFS of 18.9 months (8.7 months longer than standard EGFR-TKI) and a median OS of 38.6 months (6.8 months longer than standard EGFR-TKI). Although the OS converged at 48 months, nearly crossing-over with the standard EGFRTKI, osimertinib still demonstrated a better prognosis for advanced NSCLC patients. However, FLAURA did not provide information regarding concomitant mutation, and whether patients with $E G F R+T P 53$ would benefit more from osimertinib still lacks evidence. Thus, we performed organoid culture to examine the sensitivity of multiple EGFR-TKIs. Data analysis showed that osimertinib has the highest sensitivity, followed by first-generation EGFRTKIs, and second-generation EGFR-TKIs show the lowest sensitivity. In conclusion, it is reasonable to use osimertinib as first-line treatment in this patient. However, extending the application of organoid drug-sensitive tests to all clinical circumstances requires randomized clinical trial validation.

\section{Department of Thoracic Surgery}

Early recurrence in a stage IA LADC patient is a rare condition characterized by newly reported lung nodules or metastases in lymph nodes or other organs with the same genetic characteristics. A recurrence rate of $12.5-12.6 \%$ was reported in both retrospective and prospective studies $(10,25)$. Among those who had a recurrent disease, median time to recurrence was 15 months (25). Median diseasefree survival (DFS) was 47.2 months, and 2-year DFS rate was $93 \%(10)$. Due to its low recurrence rate and the treatment side effects, some surgeons insist on treatwhen-recur, but others support the early application of adjuvant therapy due to the poor prognosis of these stage 
IA patients after recurrence. Therefore, it is crucial to identify those patients with a high recurrence risk and to apply the most suitable adjuvant therapy as early as possible (10). Besides pathological features and serum tumor biomarkers (9), early genetic testing may aid earlystage LADC risk evaluation. Small panels covering classic driver genes tend to be cost-effective and an increasing trend of applying small panel in early-stage LADC has been observed. However, the predictive value of driver mutations still needs investigation $(13,14)$. The coexistence of TP53 has been discussed as a poor prognostic predictor in LADCs harboring EGFR mutations and might be one of the factors that contributes to the early recurrence of the tumor (15-17). Despite this, TP53 is not included in the National Comprehensive Cancer Network (NCCN) clinical practice guideline of recommended molecular biomarkers. Thus, a panel of NGS including driver genes and TP53 might be needed in the predictive evaluation of early-stage NSCLC.

The application of adjuvant therapy in the overall management is a complement to curative resection, but the value of adjuvant therapy in early-stage NSCLC has not been widely recognized. For stage I patients with high-risk pathological features of recurrence, adjuvant chemotherapy can be beneficial and monotherapy is enough for these patients (10). Very few phase III clinical trials of adjuvant chemotherapy have included stage IA NSCLC. This may result from patients' fear toward adverse events brought by chemotherapy and clinical ethical challenges, as these subgroups of NSCLC patients show a 5 -year OS of greater than $90 \%$ (1). To improve the risk-benefit balance, more attention has been drawn to target adjuvant therapy. However, it is still controversial whether adjuvant therapy should be applied to stage IA patients. In EGFRpositive NSCLC patients, the single-arm phase II trial of post-operative adjuvant erlotinib (SELECT) showed that 2 -year DFS was improved compared to historical data. SELECT included 13 patients with stage IA disease and 32 with stage IB disease. The stage I patients achieved a 2 -year DFS greater than $96 \%$, but DFS converged after 3 years (26). None of the recent phase III randomized clinical trials on EGFR-TKI have included stage IA patients (27-30). Whether TKI adjuvant therapy would lead to resistance or early recurrence is still being validated through preclinical and real-world data.

The selection of the treatment regimen for this patient included 2 complementary strategies, the first was genedrug correlation, and the second was black box testing based on a $3 \mathrm{D}$ in vitro model of PDO. Due to the existence of $E G F R^{\mathrm{L} 858 \mathrm{R}}$, the patient was responsive to EGFR-TKIs. The PDO strategy helped to evaluate which EGFR-TKI could exert better efficacy.

\section{Three questions for further discussion}

Question 1: As indicated by the above, LADC that harbors EGFR + TP53 mutations has worse prognosis than single-EGFR-mutated LADC. However, it still remains controversial to recommend adjuvant therapy for stage I patients, especially in those whose lesions are smaller than $4 \mathrm{~cm}$ in diameter. Ultimately, the question is if adjuvant therapy should be used in stage I LADC patients with high risk molecular diagnosis, and which of EGFR-TKI or chemotherapy should be chosen as adjuvant therapy based on their durability and toxicity

\section{Expert opinion 1: Dr. Daniel C. Christoph}

In case of proven pleural invasion and/or lymphatic or venous invasion adjuvant treatment should be discussed with the patient and the results of a large Japanese retrospective multicenter study presented at the last ASCO annual meeting should be explained. If the patient has no serious comorbidities (Charlson comorbidity index), I recommend adjuvant chemotherapy within 2 months after the initial resection, because targeted agents have not yet proven any OS benefit in the adjuvant setting in phase 3 prospective clinical trials. But if the postoperatively tested serum levels of tumors markers were within the regular range and no pathological signs of invasion of the primary tumor could be demonstrated, I would perform surveillance only.

\section{Expert opinion 2: Dr. Antonio Passaro}

The role of adjuvant chemotherapy in NSCLC is established for resected disease with nodal involvement (either N1 or N2 levels), thought the achieved benefit is limited, accounting of about $4-5 \%$ in absolute increase in 5 years of survival. Cisplatin-based chemotherapy is worldwide approved as the standard adjuvant therapy, with the combination with cisplatin plus vinorelbine, that is the recommended standard worldwide.

For patients with N0, adjuvant treatment might be considered, with a lower level of evidence, for tumors larger than $4 \mathrm{~cm}$ in diameter.

At the present time, 8 clinical trials evaluated the role of EGFR-TKI in adjuvant setting, including patients 
with resected stage IA-IIIA harbouring EGFR mutations, without OS benefit (31).

\section{Expert opinion 3: Dr. Cesare Gridelli}

In my opinion there is no indication to adjuvant chemotherapy or however to any adjuvant treatment for patients with stage $I$ and $T$ less than $4 \mathrm{~cm}$. In particular, in lung adjuvant cisplatin evaluation (LACE) metaanalysis the use of adjuvant chemotherapy in stage I was even detrimental with worse survival as compared to the observational control arm. The role of adjuvant chemotherapy for stage $\mathrm{I}$ with $\mathrm{T}$ more or equal $4 \mathrm{~cm}$ is controversial and optional.

In case of $E G F R^{+}$patients, even with concurrent TP53positive mutation, adjuvant targeted therapy is not yet recommended because we are waiting for the results of ongoing phase III randomized trials with the best hope for the osimertinib study.

\section{Expert opinion 4: Dr. Tomoyuki Hishida}

My current answer is no. As the authors mention, no positive results about the survival benefit of platinum-based adjuvant chemotherapy have been demonstrated for p-stage IA disease. Although previous CALGB 9633 and JBR-10 trials indicated potential survival benefit in patients with p-stage IB tumors that are $4 \mathrm{~cm}$ or larger (p-stage IIA in the present TNM $8^{\text {th }}$ edition), in a subset of analyses, no OS benefit was shown for patients with p-stage IB tumors less than $4 \mathrm{~cm}$, and p-IA disease patients were excluded from both trials. The LACE meta-analysis showed adverse effects of chemotherapy for the p-stage IA group with a hazard ratio (HR) of 1.40 (95\% CI: 0.95-2.06) (6). Adjuvant trials using EGFR-TKIs targeted more advanced p-stage IB, II, and IIIA, but did not demonstrate any OS benefit in these populations $(27,32)$. Tegafur-uracil (UFT) has shown mild survival benefits for p-stage IA NSCLC $(>2 \mathrm{~cm})$ with a HR of 0.62 (95\% CI: 0.42-0.90) in a meta-analysis of 6 Japanese trials (33). However, I think the benefits of UFT are very limited for high-risk patients. We feel tempted to use adjuvant therapy for p-stage IA patients with high-risk biological signatures; however, there is still no rationale for administering adjuvant therapy. Further investigations are needed to identify reasonable biomarkers to select good candidates for adjuvant treatment that will contribute towards prolonging patient survival.
Question 2: Considering the toxicity of adjuvant chemotherapy and economic burden of adjuvant target therapy, how should early-stage LADC patients be identified who have a high risk of relapse for administration of adjuvant therapy with a target? Also, is it rational to use NGS to test the genetic mutation in early-stage NSCLC?

\section{Expert opinion 1: Dr. Daniel C. Christoph}

For the identification of these patients clinical/pathological factors of the patients need to be known: smoking status (EGFR-diagnostics should be performed in never smokers and light smokers) and proof of signs of invasion resp. aggressiveness of the primary tumor (please see above). There is no rationale to use NGS for every patient, but for patients with a higher suspicion of suffering from an EGFRmutated lung cancer I recommend mutational diagnostics.

\section{Expert opinion 2: Dr. Antonio Passaro}

The identification of high-risk factors (e.g., visceral pleural invasion, as lymphatic permeation or vascular invasion), for resected NSCLC, should be highly useful for a better patient's selection to improve survival outcome (34).

The use of NGS in early stages it is highly debated, considering cost and international guidelines, that confirmed no target agents is approved in early stage. Nowadays, the role EGFR mutations in adjuvant setting it is under evaluation and the survival results of the phase III randomized trial, ADAURA [NCT02511106], will be released very soon.

In this trial, patients with resected NSCLC in stage IB, II and IIIA harboring common EGFR mutations, received osimertinib or placebo for a treatment duration of up to three years, with the primary point of DFS. Based on the preliminary data, not yet presented, the trial results will be unblinded early following a recommendation from an Independent Data Monitoring Committee (IDMC) based on its determination of overwhelming efficacy.

\section{Expert opinion 3: Dr. Cesare Gridelli}

To date is no evidence-based to do in clinical practice NGS for identify early-stage high risk patients. First, because we have no evidence that the presence of any tumor genetic alteration can drive adjuvant therapy. Second, the presence of any mutation or gene fusion or however genetic alteration can drive an adjuvant targeted 
therapy in clinical practice.

\section{Expert opinion 4: Dr. Tomoyuki Hishida}

I think the use of NGS is useful for detecting potential druggable driver gene abnormalities for targeted therapy. However, targeted therapy is usually cytostatic and not cytotoxic. Previous adjuvant EGFR-TKI trials have shown prolonged recurrence-free survival, but no OS benefit in patients with p-stage IB-IIIA NSCLC with activated EGFR mutations $(27,32)$. In my opinion, it is not too late to use targeted therapy, even if we start to use it at the time of recurrence. It remains unknown whether early use of targeted therapy as an adjuvant treatment is beneficial for prolonging patient survival and enhancing a rate of cure.

Question 3: In our case, organoids were cultured as a preclinical model for drug-sensitivity screening tests, the results of which met our expectations and facilitated the selection of treatment. However, more evidence needs to be collected from large-scale clinical trials focusing on the concordance of organoid drug sensitivity and clinical response. Despite the current evidence summarized in the introduction, it still remains unclear if organoids will play a more important role in future personalized treatment selection

\section{Expert opinion 1: Dr. Daniel C. Christoph}

There might be a role of organoids if the primary tumor shows concomitant genetic mutations with different treatment options. During the period of postoperative convalescence, drug sensitivity might be tested and the results might guide the selection of the most effective systemic treatment.

\section{Expert opinion 2: Dr. Antonio Passaro}

The role of preclinical model to test drug-sensitivity is highly potential, especially in the era of precision medicine. Lung cancer showed high genetic and phenotypic heterogeneity. Organoid evaluation should be useful and interesting to improve our knowledge on biological alteration (genomic and genetic) in early setting of lung cancer.

The next step of precision cancer medicine it will be to predict patient-specific drug responses through in vitro patient-specific drug trials.

\section{Expert opinion 3: Dr. Cesare Gridelli}

Drug-sensitivity tests should be prospectively validated and object of future phase III randomized trials. To date there is no recommendation even for the chemotherapeutic drugsensitivity test already tested in the past. In conclusion, to date there is no role for any kind of drug-sensitivity test in clinical practice.

\section{Expert opinion 4: Dr. Tomoyuki Hishida}

It is an exciting avenue to utilize organoids as a personalized in vitro model to predict response to anticancer therapy. In this case, osimertinib was identified as the treatment of choice based on the results from organoids. However, osimertinib has been known to be a key drug and the 1stline therapy for LADCs with EGFR mutations (Ex19 del or L858R) (35), in spite of the lacking data for LADCs with $E G F R+T P 53$ co-mutations, and whether it is a good candidate, can be decided without the use of an organoid model. In my opinion, the clinical benefit of organoids may be limited for LADC with driver gene abnormalities and the corresponding targeted therapies. Organoid models may pose potential benefits for LADC without driver gene abnormalities for predicting response toward conventional cytotoxic regimens and immune checkpoint inhibitors. Further studies are required to identify good targets of organoid models for the treatment of advanced NSCLC.

\section{Conclusions}

For stage I NSCLC patients with nodules less than $4 \mathrm{~cm}$, no current evidence supports the use of adjuvant therapy nor genetic testing is recommended. However, organoid, an in vitro personalized pre-clinical model, is highly potential for drug sensitive test in precision medicine. Further prospective investigations and clinical validations are needed to establish the feasibility of organoids.

\section{Acknowledgments}

The authors would like to thank Huihui Xu for her contribution to clinical information collection of this research.

Funding: This work was supported by the (I) Beijing Natural Science Foundation (7182132); (II) Special Data Service for Oncology, The National Population and Health Scientific Data Sharing Platform (NCMI-ABD02-201809; NCMI-YF02N-201906), Supported by Ministry of Science and Technology of the People's Republic of China (MOST); (III) Beijing Dongcheng District Science and Technology Commission Excellent Talents Project 
(2018-DCQYXRCXM-012); (IV) Beijing Students' Platform for Innovation and Entrepreneurship Training Program (2019zlgc0629); (V) National Key Research and Development Program of China Grant (2016YFC0901500).

\section{Footnote}

Reporting Checklist: The authors have completed the CARE reporting checklist. Available at http://dx.doi.org/10.21037/ jtd-20-1882

Conflicts of Interest: All authors have completed the ICMJE uniform disclosure form (available at http://dx.doi. org/10.21037/jtd-20-1882). ZJ reports grants from Beijing Students' platform for innovation and entrepreneurship training program, during the conduct of the study. ZC reports personal fees from Accurate International Biotechnology Co. Ltd, during the conduct of the study. $\mathrm{MH}$ reports personal fees from Accurate International Biotechnology Co. Ltd., during the conduct of the study. YY reports personal fees from Accurate International Biotechnology Co. Ltd, during the conduct of the study. HHZ reports personal fees from Burning Rock Biotechnology Co. Ltd., during the conduct of the study. JS reports personal fees from Burning Rock Biotechnology Co. Ltd., during the conduct of the study. DCC reports personal fees, non-financial support and other from AstraZeneca, personal fees, non-financial support and other from Bayer, personal fees, non-financial support and other from Boehringer-Ingelheim, personal fees, non-financial support and other from Bristol-Myers Squibb, personal fees, non-financial support and other from Chugai, personal fees, non-financial support and other from Merck, Sharp \& Dohme, personal fees, non-financial support and other from Novartis, personal fees, non-financial support and other from Pfizer, personal fees, non-financial support and other from Roche, personal fees, non-financial support and other from Takeda, outside the submitted work. AP reports personal fees from Roche, personal fees from BMS, personal fees from MSD, personal fees from Lilly, personal fees from Dako, personal fees from Astra Zeneca, outside the submitted work, and he serves as an unpaid editorial board member of Journal of Thoracic Disease from Aug 2019 to Jul 2021. CG reports personal fees from Astra Zeneca, grants and personal fees from MSD, personal fees from BMS, personal fees from Roche, personal fees from Menarini, personal fees from Novartis, outside the submitted work. NL reports grants from Beijing Natural
Science Foundation, grants from Ministry of Science and Technology of the People's Republic of China (MOST), grants from Chinese Academy of Medical Sciences Young Medical Talent Award Fund, grants from Beijing Students' platform for innovation and entrepreneurship training program, grants from National Key Research and Development Program of China Grant, during the conduct of the study. The other authors have no conflicts of interest to declare.

Ethical Statement: The authors are accountable for all aspects of the work in ensuring that questions related to the accuracy or integrity of any part of the work are appropriately investigated and resolved. All procedures performed in studies involving human participants were in accordance with the Declaration of Helsinki (as revised in 2013). Written informed consent was obtained from the patient for publication of this manuscript and any accompanying images.

Open Access Statement: This is an Open Access article distributed in accordance with the Creative Commons Attribution-NonCommercial-NoDerivs 4.0 International License (CC BY-NC-ND 4.0), which permits the noncommercial replication and distribution of the article with the strict proviso that no changes or edits are made and the original work is properly cited (including links to both the formal publication through the relevant DOI and the license). See: https://creativecommons.org/licenses/by-nc-nd/4.0/.

\section{References}

1. Deslypere G, Gullentops D, Wauters E, et al. Immunotherapy in non-metastatic non-small cell lung cancer: Can the benefits of stage IV therapy be translated into earlier stages? Ther Adv Med Oncol 2018;10:1758835918772810.

2. Choi JI. Medically inoperable stage I non-small cell lung cancer: best practices and long-term outcomes. Transl Lung Cancer Res 2019;8:32-47.

3. Sawabata N, Miyaoka E, Asamura H, et al. Japanese lung cancer registry study of 11,663 surgical cases in 2004: demographic and prognosis changes over decade. J Thorac Oncol 2011;6:1229-35.

4. Mitsudomi T, Suda K, Yatabe Y. Surgery for NSCLC in the era of personalized medicine. Nat Rev Clin Oncol 2013;10:235.

5. Milano MT, Kong FM, Movsas B. Stereotactic body 
radiotherapy as salvage treatment for recurrence of nonsmall cell lung cancer after prior surgery or radiotherapy. Transl Lung Cancer Res 2019;8:78-87.

6. Pignon JP, Tribodet H, Scagliotti GV, et al. Lung adjuvant cisplatin evaluation: a pooled analysis by the LACE Collaborative Group. J Clin Oncol 2008;26:3552-9.

7. Kris MG, Gaspar LE, Chaft JE, et al. Adjuvant systemic therapy and adjuvant radiation therapy for stage I to IIIA completely resected non-small-cell lung cancers: American Society of Clinical Oncology/Cancer Care Ontario Clinical Practice Guideline Update. J Clin Oncol 2017;35:2960-74.

8. Vansteenkiste J, De Ruysscher D, Eberhardt WEE, et al. Early and locally advanced non-small-cell lung cancer (NSCLC): ESMO Clinical Practice Guidelines for diagnosis, treatment and follow-up $\dagger$. Ann Oncol 2013;24:vi89-98.

9. Der SD, Sykes J, Pintilie M, et al. Validation of a histology-independent prognostic gene signature for early-stage, non-small-cell lung cancer including stage IA patients. J Thorac Oncol 2014;9:59-64.

10. Park HL, Yoo IR, Boo SH, et al. Does FDG PET/ CT have a role in determining adjuvant chemotherapy in surgical margin-negative stage IA non-small cell lung cancer patients? J Cancer Res Clin Oncol 2019;145:1021-6.

11. Zhou C, Wu YL, Chen G, et al. Erlotinib versus chemotherapy as first-line treatment for patients with advanced EGFR mutation-positive non-small-cell lung cancer (OPTIMAL, CTONG-0802): a multicentre, open-label, randomised, phase 3 study. Lancet Oncol 2011;12:735-42.

12. $\mathrm{Wu} \mathrm{YL}, \mathrm{Zhou} \mathrm{C}, \mathrm{Hu} \mathrm{CP}$, et al. Afatinib versus cisplatin plus gemcitabine for first-line treatment of Asian patients with advanced non-small-cell lung cancer harbouring EGFR mutations (LUX-Lung 6): an open-label, randomised phase 3 trial. Lancet Oncol 2014;15:213-22.

13. Goldstraw P, Chansky K, Crowley J, et al. The IASLC Lung Cancer Staging Project: Proposals for Revision of the TNM Stage Groupings in the Forthcoming (Eighth) Edition of the TNM Classification for Lung Cancer. J Thorac Oncol 2016;11:39-51.

14. Janjigian YY, Park BJ, Zakowski MF, et al. Impact on disease-free survival of adjuvant erlotinib or gefitinib in patients with resected lung adenocarcinomas that harbor EGFR mutations. J Thorac Oncol 2011;6:569-75.

15. Vanderlaan PA, Rangachari D, Mockus SM, et al. Mutations in TP53, PIK3CA, PTEN and other genes in
EGFR mutated lung cancers: Correlation with clinical outcomes. Lung Cancer 2017;106:17-21.

16. Maiga AW, Deppen SA, Mercaldo SF, et al. Assessment of Fluorodeoxyglucose F18-Labeled Positron Emission Tomography for Diagnosis of High-Risk Lung Nodules. JAMA Surgery 2018; 153:329.

17. Labbé C, Cabanero M, Korpanty GJ, et al. Prognostic and predictive effects of TP53 co-mutation in patients with EGFR-mutated non-small cell lung cancer (NSCLC). Lung Cancer 2017;111:23-9.

18. Ramalingam SS, Gray JE, Ohe Y, et al. Osimertinib vs comparator EGFR-TKI as first-line treatment for EGFRm advanced NSCLC (FLAURA): Final overall survival analysis. Ann Oncol 2019;30:v851-v934.

19. Clevers H. Modeling development and disease with organoids. Cell 2016;165:1586-97.

20. Bregenzer ME, Horst EN, Mehta P, et al. Tumor modeling maintains diverse pathology in vitro. Ann Transl Med 2019;7:S262.

21. Sato T, Stange DE, Ferrante M, et al. Long-term expansion of epithelial organoids from human colon, adenoma, adenocarcinoma, and Barrett's epithelium. Gastroenterology 2011;141:1762-72.

22. Weeber F, van de Wetering M, Hoogstraat $M$, et al. Preserved genetic diversity in organoids cultured from biopsies of human colorectal cancer metastases. Proc Natl Acad Sci U S A 2015;112:13308-11.

23. Behjati S, Huch M, van Boxtel R, et al. Genome sequencing of normal cells reveals developmental lineages and mutational processes. Nature 2014;513:422.

24. Li X, Francies HE, Secrier M, et al. Organoid cultures recapitulate esophageal adenocarcinoma heterogeneity providing a model for clonality studies and precision therapeutics. Nat Commun 2018;9:2983.

25. Zhang Y, Sun Y, Xiang J, et al. A clinicopathologic prediction model for postoperative recurrence in stage Ia non-small cell lung cancer. J Thorac Cardiovasc Surg 2014;148:1193-9.

26. Pennell NA, Neal JW, Chaft JE, et al. SELECT: A Phase II Trial of Adjuvant Erlotinib in Patients With Resected Epidermal Growth Factor Receptor-Mutant Non-SmallCell Lung Cancer. J Clin Oncol 2019;37:97-104.

27. Kelly K, Altorki NK, Eberhardt WEE, et al. Adjuvant Erlotinib Versus Placebo in Patients With Stage IBIIIA Non-Small-Cell Lung Cancer (RADIANT): A Randomized, Double-Blind, Phase III Trial. J Clin Oncol 2015;33:4007-14.

28. Li N, Ou W, Ye X, et al. Pemetrexed-carboplatin adjuvant 
chemotherapy with or without gefitinib in resected stage IIIA-N2 non-small cell lung cancer harbouring EGFR mutations: a randomized, phase II study. Ann Surg Oncol 2014;21:2091-6.

29. Zhong WZ, Wang Q, Mao WM, et al. Gefitinib versus vinorelbine plus cisplatin as adjuvant treatment for stage II-IIIA (N1-N2) EGFR-mutant NSCLC (ADJUVANT/ CTONG1104): a randomised, open-label, phase 3 study. Lancet Oncol 2018;19:139-48.

30. Wu YL, Herbst RS, Mann H, et al. ADAURA: Phase III, Double-blind, Randomized Study of Osimertinib Versus Placebo in EGFR Mutation-positive Early-stage NSCLC After Complete Surgical Resection. Clin Lung Cancer 2018;19:e533-6.

31. Attili I, Passaro A, Spaggiari L, et al. Adjuvant EGFR TKIs in NSCLC harboring EGFR mutations: looking for a consensus way. Ann Transl Med 2020. doi: 10.21037/ atm.2020.04.35.

Cite this article as: Jia Z, Wang Y, Cao L, Wang Y, Song Y, Yang X, Bing Z, Cao Z, Liu P, Zhang S, Chen Z, Huang M, Yu Y, Han-Zhang H, Song J, Christoph DC, Passaro A, Gridelli C, Hishida T, Liang N, Li S. First-line treatment selection with organoids of an EGFRm + TP53m stage IA1 patient with early metastatic recurrence after radical surgery and follow-up. J Thorac Dis 2020;12(7):3764-3773. doi: 10.21037/jtd-20-1882
32. Goss GD, O'Callaghan C, Lorimer I, et al. Gefitinib versus placebo in completely resected non-small-cell lung cancer: results of the NCIC CTG BR19 study. J Clin Oncol 2013;31:3320-6.

33. Hamada C, Tsuboi M, Ohta M, et al. Effect of postoperative adjuvant chemotherapy with tegafur-uracil on survival in patients with stage IA non-small cell lung cancer: an exploratory analysis from a meta-analysis of six randomized controlled trials. J Thorac Oncol 2009;4:1511-6.

34. Tsutani Y, Imai K, Ito H, et al. Adjuvant chemotherapy for pathological stage I non-small cell lung cancer with highrisk factors for recurrence: A multicenter study. J Clin Oncol 2019;37:abstr 8500.

35. Ramalingam SS, Vansteenkiste J, Planchard D, et al. Overall survival with osimertinib in untreated, EGFR-mutated advanced NSCLC. N Engl J Med 2020;382:41-50. 


\section{Methods}

\section{Patient-derived tumor organoid culture}

The primary organoids were established using previously described culture methods with modification (1). Briefly, solid lung cancer tissue was rinsed by $10 \mathrm{~mL}$ HBSS containing antibiotics, then minced by sterilized surgical scissors, and digested in $5 \mathrm{~mL}$ of $5 \mathrm{mg} / \mathrm{mL}$ collagenase type II (Invitrogen) in DMEM/F12 for about $4 \mathrm{~h}$ at $37^{\circ} \mathrm{C}$ with gentle shaking and intermittent pipetting. The digested tissue suspension was strained over a $70 \mu \mathrm{m}$ filter. The strained suspension was centrifuged at $300 \mathrm{rcf}$, and red blood cells were lysed using lysis buffer (00443357, Invitrogen eBioscience) for $5 \mathrm{~min}$. Dissociated cells were washed, counted, and resuspended in Matrigel basement membrane Matrix (Corning, 356235). Next, $30 \mu \mathrm{L}$ drops of Matrigel-cell suspension were allowed to solidify on pre-warmed $60 \mathrm{~mm}$ culture plates in a $37{ }^{\circ} \mathrm{C}$ and $5 \% \mathrm{CO}_{2}$ cell culture incubator for 30 min. Organoids were cultured in Advanced DMEM/F12 (Thermo Fisher Scientific), supplemented with the additives listed in Table S1. Upon completed gelation, culture medium was added to each plate. Medium was refreshed every 4 days (21).

\section{Organoid drug response assay}

Organoids were harvested using $1 \times$ TrypLe (Gibco) and dissociated to small clusters. Between 100 and 500 clusters were plated in $40-\mu \mathrm{L} 5 \%$ Matrigel/organoid culture medium in a 384-well plate (Corning) in triplicate. Clear bottom 384-well plates were coated with $10-\mu \mathrm{L}$ collagen (Thermo Fisher) prior to plating cells. Then, $48 \mathrm{~h}$ after plating, a concentration dilution series of each compound $(50,10,2,0.4,0.08,0.016 \mu \mathrm{M})$ was dispensed using liquid-handling robotics, and cell viability was assayed using CellTiter-Glo (Promega) after $4 \mathrm{~d}$ of drug incubation. Results were normalized to vehicle controls. Data analyses were performed using GraphPad Prism 7.0 software, and the values of IC50 were calculated by applying nonlinear regression (curve fit).

Table S1 Organoid culture additives

\begin{tabular}{|c|c|c|c|}
\hline Additive & Supplier & Catalogue \# & Concentration \\
\hline EGF & PeproTech & AF-100-15 & 50 ng/mL \\
\hline FGF-10 & PeproTech & $100-26$ & 20 ng/mL \\
\hline FGF-basic & PeproTech & $100-10 B$ & $1 \mathrm{ng} / \mathrm{mL}$ \\
\hline A-83-01 & Sigma & SML0788 & $500 \mathrm{nM}$ \\
\hline SB202190 & Selleck & S1077 & $10 \mu \mathrm{M}$ \\
\hline Nicotinamide & Sigma & 72340 & $10 \mathrm{mM}$ \\
\hline PGE2 & Tocris & 2296 & $1 \mu \mathrm{M}$ \\
\hline HEPES & Sigma & H6147 & $10 \mathrm{mM}$ \\
\hline GlutaMAX & Gibco & 35050061 & $1 x$ \\
\hline B27 & Gibco & 17504044 & $1 \times$ \\
\hline N2 & Gibco & 17502048 & $1 \times$ \\
\hline
\end{tabular}

R-Spondin 1 and Noggin conditioned media were prepared in house from the culture media of Rspo1 cell and the noggin cDNA transfected HEK-293 cell following the methods described before $(36,37)$. 


\section{References}

36. Degese S, Benton G. Production of R-Spondin 1 conditioned medium. Available online: http://www.interchim.com/forum/ production-of-r-spondin-1-conditioned-medium-t1448.html

37. Wang Y, Ahmad AA, Sims CE, et al. In vitro generation of colonic epithelium from primary cells guided by microstructures. Lab Chip 2014;14:1622-31. 